\title{
Evaluation de l'agar, matériau solide présentant des caractéristiques acoustiques équivalentes à celles de l'eau
}

\author{
A. BOUAKKAZ, C. CACHARD et G. GIMENEZ
}

CREATIS, URA 1216 du CNRS, INSA 502, 69621 Villeurbanne cedex, France

\begin{abstract}
RESUME: Le but de cette étude est de déterminer les caractéristiques acoustiques de l'agar, un matériau acoustiquement proche de l'eau. L'avantage de l'agar est que c'est un matériau solide, ce qui élargit son domaine d'application. Avec de telles propriétés, l'agar peut être utilisé comme conteneur de solutions liquides lors de leur caractérisation. De plus, son utilisation comme milieu de couplage ne nécessite pas un contact physique entre la cible et l'émetteur. Son utilisation peut s'étendre également au domaine médical (échographie) où il peut servir comme fantôme pour des évaluations ultrasonores des tissus.
\end{abstract}

\begin{abstract}
The aim of our work is to determine the acoustic caracteristics of agar, a material acoustically equivalent to water. The advantage of the use of agar is that it presents properties very neighbouring to those of water, and it's a solid material. Such properties make its areas very diversified and include medicine domain (echography) where it's used as a phantom. It's also used as an insulator to eliminate echos which may be important with respect to informationel echos. So, it's too necessary to know his acoustic behaviour by determining the attenuation coefficient, the speed of sound and the acoustic impedance.
\end{abstract}

\section{INTRODUCTION}

Lors de l'évaluation des propriétés acoustiques de certains matériaux ou de certains objets, on est confronté à un choix: soit maintenir l'objet en suspension dans l'eau en prenant le risque de modifier son comportement acoustique, soit l'enfermer dans un conteneur mais dans ce cas les échos d'interface dus au conteneur peuvent devenir prépondérants dans le signal rétrodiffusé. Pour palier à cette difficulté, il est possible d'utiliser un conteneur solide dont les propriétés sont proches de celles de l'eau. Un matériau qui semble remplir ces conditions est l'agar [1]. C'est un composé polygalactoside extrait d'une algue marine, et qui a une structure solide sous certaines conditions et avec une préparation adéquate. De ce fait, il parait nécessaire de connaitre le comportement acoustique de ce matériau en quantifiant ses paramètres à savoir: le coefficient d'atténuation, la vitesse de propagation et l'impédance acoustique. Signalons que l'agar est également utilisé comme fantôme en échographie car sa structure est proche de celle des tissus humains.

\section{PREPARATION}

Pour obtenir un milieu solide d'agar, un mélange de 30 à $40 \mathrm{~g}$ de poudre d'agar par litre d'eau est chauffé à $80^{\circ} \mathrm{C}$ durant une heure, tout en gardant le mélange en agitation permanente. Le mélange devient translucide, alors il est versé dans un moule de forme convenable. Le refroidissement s'effectue à la température ambiante. La structure obtenue forme un bloc solide d'agar qui doit être conservé dans l'eau pour qu'il garde au mieux ses propriétés. 


\section{MESURES ULTRASONORES:}

L'une des techniques généralement utilisée pour l'évaluation ultrasonore des matériaux est la technique de transmission. L'interprétation de l'onde radio fréquence transmise à travers l'échantillon permet l'extraction de deux paramètres, en l'occurrence le temps de vol et l'amplitude de l'écho. La mesure du temps de vol est liée à une mesure de vitesse et la mesure de l'amplitude est liée à celle de l'atténuation.

Le système de mesure (figure 1) comprend un émetteur $\mathrm{E}$ et un récepteur $\mathrm{R}$ entre lesquels est interposé le bloc d'agar, le tout est immergé dans un bain thermostaté à $20^{\circ} \mathrm{C}$. L'émetteur et le récepteur sont placés face à face sur deux supports solidaires. Le système comprend également un générateur d'impulsions PANAMETRICS 5052 PR qui délivre des impulsions d'énergie et de durée réglables, un oscilloscope numérique LECROY 9401 relié à un micro-ordinateur pour l'acquisition et le traitement de données.

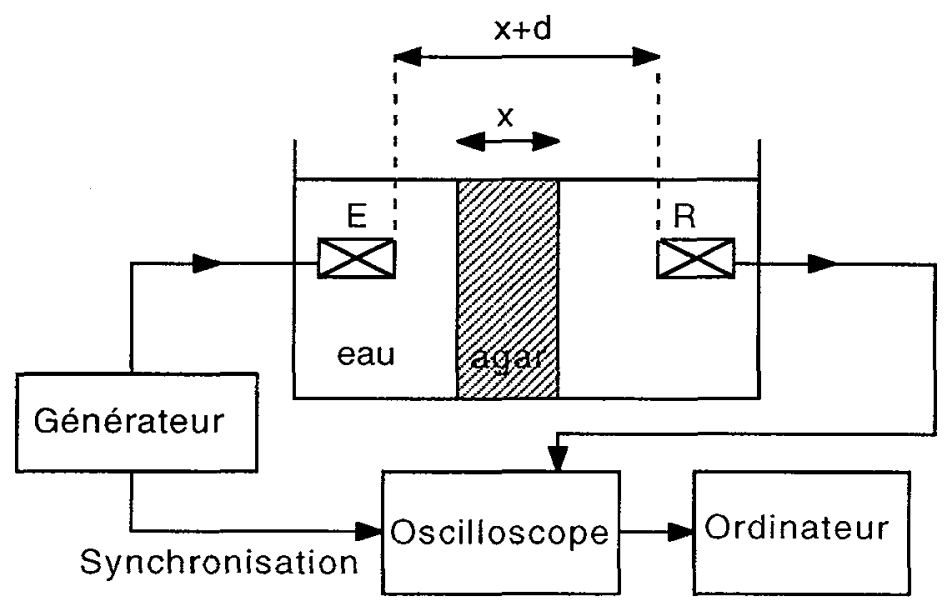

Figure 1: dispositif de mesure.

Les mesures sont effectuées à différentes fréquences avec des traducteurs à bandes étroites. On a repris également ces mesures en utilisant deux traducteurs large bande pour couvrir la gamme 0,5-22 $\mathrm{MHz}$, leurs fréquences centrales étant 10 et $20 \mathrm{MHz}$. Cette dernière permet des mesures continues en fonction de la fréquence.

L'atténuation est déterminée à partir de deux mesures, l'une dite mesure de référence, est effectuée pour déterminer la contribution du système de mesure ( la fonction d'appareil). Elle est représentée par le signal transmis uniquement dans l'eau en l'absence de l'échantillon test. La deuxième mesure est effectuée en présence du bloc d'agar. Elle contient en plus de la fonction d'appareil, la contribution de l'échantillon. Ainsi, l'atténuation est calculée à partir d'une déconvolution numérique en utilisant une division spectrale. A noter que la déconvolution n'est effectuée que dans la bande de fréquence définie à $-20 \mathrm{~dB}$.

\section{RESULTATS}

\subsection{Mesures d'atténuation}

Comme exposé ci-dessus, l'atténuation est déterminée à partir du rapport spectral des spectres des signaux transmis avec et en l'absence du bloc d'agar. Cette atténuation en fonction de la fréquence est représentée sur la figure 2 . On remarque que pour des fréquences inférieures à $3 \mathrm{MHz}$, l'atténuation est très faible. L'agar peut alors être considéré dans cette gamme de fréquence comme transparent aux ultrasons. Au delà de cette fréquence, l'atténuation augmente. Sur la même fïgure est tracé également l'atténuation du même bloc, mais mesurée avec les différents traducteurs (0,2-0,5-1-2 et $5 \mathrm{MHz})$. On a ainsi, 5 segments de courbes qui se superposent assez bien sur la courbe tracée avec les deux traducteurs large bande. 


$$
\mathrm{A}(\mathrm{x}) / \mathrm{A}(0)=\exp \left(-\alpha \mathrm{f}^{\mathrm{n}} \mathrm{x}\right)
$$

avec: $\quad A(0)$ : amplitude à l'origine;

$\mathrm{A}(\mathrm{x})$ : amplitude à la distance $\mathrm{x}$;

$\alpha$ : coefficient d'atténuation;

n: exposant;

$\mathrm{x}$ : distance (épaisseur du bloc);

La représentation du terme $\alpha \mathrm{f}^{\mathrm{n}} \mathrm{x}$ en fonction du carré de la fréquence (figure 3 ) donne une droite. C'est à dire que comme pour l'eau, l'exposant $\mathrm{n}$ vaut 2 et la pente de cette droite donne le coefficient d'atténuation $\alpha_{\text {agar }}=55.10^{-15} \mathrm{~dB} \cdot \mathrm{Hz}^{-2} \cdot \mathrm{m}^{-1}$. L'agar présente donc un coefficient très faible et proche de celui de l'eau $\left(\alpha_{\text {eau }}=22 \cdot 10^{-15} \mathrm{~dB} \cdot \mathrm{Hz}^{-2} \cdot \mathrm{m}^{-1}\right)$.

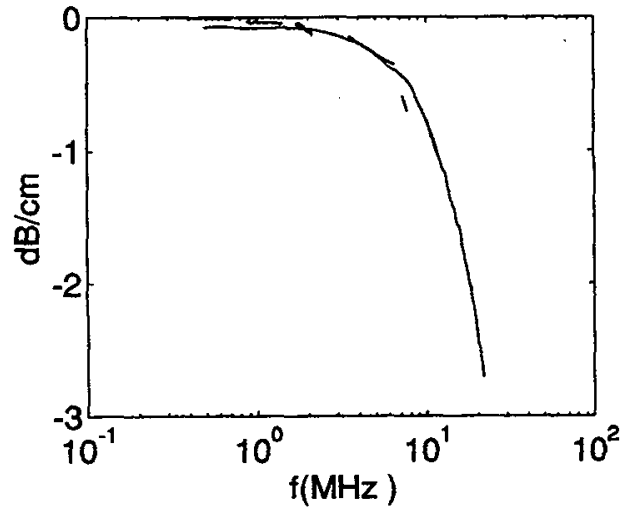

Figure 2: atténuation de l'agar en fonction de la fréquence.

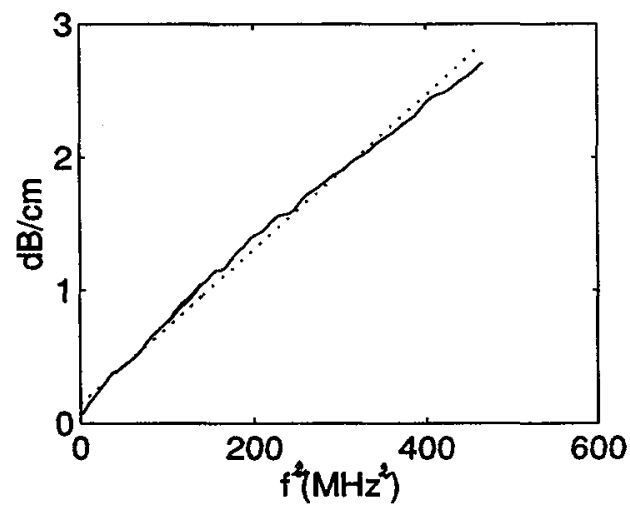

Figure 3: le terme $\alpha \mathrm{f}^{\mathrm{n}} \mathrm{x}$ en fonction du carre de la fréquence, -- courbe expérimentale, ... courbe approximée. 


\subsection{Mesures de vitesse}

Le dispositif expérimental utilisé est identique à celui utilisé et représenté sur la figgure 1, en utilisant les traducteurs non ammortis. Les mesures sont effectuées pour chacune des fréquences. La vitesse de propagation dans l'agar est mesurée en utilisant la technique de mesure relative, c'est à dire à partir de la mesure de la vitesse de propagation dans le milieu de référence (eau ). De la figure 1, on déduit:

$$
\tau=\frac{\mathrm{x}}{\mathrm{c}_{\mathrm{agar}}}+\frac{\mathrm{d}}{\mathrm{c}_{\mathrm{eau}}}
$$

avec: $\quad \mathrm{x}=$ épaisseur du bloc d'agar;

$\mathrm{d}=$ distance parcourue à travers le milieu de référence (eau);

$\tau=$ temps de vol;

Le temps de vol est déterminé soit par intercorrélation des signaux transmis avec et en l'absence du bloc, soit par transformation de Fourier. Les deux méthodes donnent des résultats identiques avec une précision de l'ordre de $0,2 \%$. De plus sur la bande de fréquence étudiée, la variation de la vitesse en fonction de la fréquence est négligeable. Pour l'agar, on obtient $c_{\mathrm{agar}}=1491 \mathrm{~m} . \mathrm{s}^{-1}$ alors que pour l'eau, $c_{\text {eau }}=1483 \mathrm{~m} \cdot \mathrm{s}^{-1}$. L'impédance acoustique de l'agar $\mathrm{Z}=\rho \mathrm{v}$ se déduit d'une mesure de densité, ce qui donne $\mathrm{Z}=1497.10^{3} \mathrm{Kg} \cdot \mathrm{m}^{-2} \cdot \mathrm{s}^{-1}$.

On présente dans le tableau 1 un récapitulatif des différents paramètres acoustiques comparant l'eau à l'agar. Ainsi, on constate une bonne similitude entre ces deux matériaux.

\begin{tabular}{|l|l|l|}
\hline & eau & agar \\
\hline$\alpha\left[\mathrm{dB} \cdot \mathrm{Hz}^{-2} \cdot \mathrm{m}^{-1}\right]$ & $22.10-15$ & $55.10^{-15}$ \\
\hline $\mathrm{c}\left[\mathrm{m} \cdot \mathrm{s}^{-1}\right]$ & 1483 & 1491 \\
\hline $\mathrm{n}$ & 2 & 2 \\
\hline $\mathrm{\rho}[\mathrm{Kg} \cdot \mathrm{m}-3]$ & 1000 & 1005 \\
\hline $\mathrm{Z}\left[\mathrm{Kg} \cdot \mathrm{m}^{-2} \cdot \mathrm{s}^{-1}\right]$ & $1483.10^{3}$ & $1497.10^{3}$ \\
\hline
\end{tabular}

Tableau 1: comparaison des paramètres acoustiques de l'eau et de l'agar.

\section{CONCLUSION}

En utilisant la technique de transmission par substitution, on a déterminé les propriétés acoustiques de l'agar, matériau solide et acoustiquement transparent. Ces propriétés étant mesurées principalement dans la gamme des fréquences, entre 0,2 et $22 \mathrm{MHz}$. Les résultats montrent que c'est un matériau possédant des caractéristiques acoustiques similaires à celles de l'eau. Ainsi, sur une large gamme de fréquences, il peut être considéré comme transparent aux ultrasons, et le fait qu'il soit solide élargit son domaine d'application. En effetet, il peut être utilisé comme conteneur solide et transparent. Les ondes ultrasonores le traversent avec un minimum d'atténuation, et les échos d'interface sont quasiment inexistant. Son domaine d'application peut comprendre aussi le domaine médical et principalement l'échographie où il peut servir comme fantôme pour des évaluations ultrasonores.

\section{REFERENCES}

[1] Chivers RC, Hill CR. "Ultrasonic attenuation in human tissue," Ultrasdound Med Biol 2:25-29, October 1975.

[2] Hirohuki Hachiya and Shigea Ohtsuki (1992). "Determination of sound speed in biological tissues based on frequency analysis of pulse response", J. Acoust. Soc. Am, Vol 92, No3, 1564-1568, September 1992. 